\title{
Exploring Challenges and Opportunities to Support People with Food Allergies to Find Safe Places for Eating Out
}

\author{
Favina Aluvathingal \\ University of Leicester \\ University Road, Le1 7RH, Leicester \\ favinaa@gmail.com
}

\author{
Nervo Verdezoto \\ University of Leicester \\ University Road, Le1 7RH, Leicester \\ nervo.verdezoto@leicester.ac.uk
}

\author{
Francisco Nunes \\ Fraunhofer Portugal AICOS \\ R. Alfredo Allen 455, Porto, Portugal \\ francisco.nunes@fraunhofer.pt
}

\begin{abstract}
Eating out can be problematic for people who have food allergies. For example, the fear of having an allergic reaction while eating out can cause anxiety and stress especially for young adults, impacting their personal and social life. To address this issue, we describe "AllergyFreeFoodie", a crowdsourced mobile application that aims to support people with food allergies in searching for safe places for eating out. We present an initial prototype together with the results of a preliminary evaluation with 6 young adults in the UK. Based on early findings, we highlight some of the challenges that our participants face in their everyday life and some opportunities for the redesign of the application to promote an allergy-friendly environment for people with food allergies.
\end{abstract}

Food Allergens. Food Allergy. Eating out. Dining out. Mobile application. Design. HCl. Crowdsourcing.

\section{INTRODUCTION}

According to the Food Standards Agency, it is estimated that over two million people in the UK have a food allergy (FSA 2016). Having a food allergy or food hypersensitivity can be described as an abnormal response or reaction to a given food often triggered by the body's immune system (Luyt et al. 2016). Food allergy can also be non-immune mediated when caused, for example, by an allergy to cow's milk and soy proteins that are rarely life threatening (Jyonouchi 2008). Allergic reactions can vary from mild, for example a skin rash, to more severe reactions such as anaphylaxis reactions, which can include gastrointestinal and respiratory symptoms (Luyt et al. 2016). While reactions triggered by the immune system take place within 2 hours after eating, non-immune allergic reactions can take sometimes up to 48-72 hours to appear (Luyt et al. 2016, nhs.uk 2016). Moreover, while any food can cause allergic reactions, the most common ones are attributed to peanuts, milk, eggs, shellfish, fish, tree nuts, wheat, soya, sesame, and kiwi fruit (Luyt et al. 2016).

A study conducted in the UK reported that around $50 \%$ of young adults (18-34 years old) are eating out more often due to the dynamic changes in work routines, increased freedom and flexibility for choosing where to eat and an increased availability of online services providing restaurant's information, enabling people to share their food experiences (Sacla 2016). Indeed, food experiences can be regarded as an important component for social bonding in different cultures. However, people living with food allergies can experience difficulties in their eating practices while eating out, which impact not only their body and daily life, but also their social experiences and engagement with others (Pitchforth et al. 2011, Begen et al. 2018).

In this work-in-progress paper, we share our initial insights towards the design of a crowdsourced mobile application that aims to support people with food allergies to find safe places for eating out. We derived an initial list of requirements by analysing existing applications listed in mobile application stores. Based on the proposed requirements, we created a low-fidelity prototype of our initial design concept, AllergyFreeFoodie, and evaluated it with 6 participants with food allergies. Participants gave initial feedback and commented on the potential benefits to support their information-seeking journey and enhance their eating out experiences.

\section{RELATED WORK}

Eating out is a problematic task for people with food allergies (Pitchforth et al. 2011). In December 2014, the European Union Food Information for Consumers Regulation (EU FIC) changed the way food businesses are expected to provide allergen information to consumers (FSA 2016). According to these regulations, every food establishment must provide information on their menus regarding 14 main allergens, including the common ones such as peanuts and gluten and less common ones like 
mustard and celery. In addition, Lee and Kwon (2011) reports some of the main factors that affect eating out experience for people with food allergies. Firstly, the potential cross contact of food with allergen(s) in the kitchen, directly, or through the contact with cooking equipment and utensils. The second factor reported is the lack of training of employees as they might not be aware of the severity and adverse effects of allergic reactions triggered by food allergens. For example, restaurant employees might not want to remove or substitute ingredients from their original recipe, because they are not aware of the impact of their decision. Last but not least, restaurant's menus can have misleading or insufficient information about the food ingredients that can be labelled as "fussy" or using the wording "might contain", increasing the risk and uncertainty of eating out experiences (Lee and Kwon, 2011).

Information and communication technologies can offer opportunities to support people with food allergies to cope with their everyday challenges. Previous work suggested using food diaries for families to keep track of when specific food was taken (Henricksen and Viller, 2012). This specific food diary exploited gamification mechanisms, visualizations, prompts, and reminders to encourage data entry. In addition, Davidson et al. (2017) explored React, a mobile application which used video-based anaphylaxis narratives to illustrate to young people how different selfmanagement choices may have different consequences. Similarly, Hernandez-Munoz et al. (2017) presents AllergieSense, a smartphone application and sensing system for emergency preparedness to manage anaphylaxis reactions. It includes a list of videos about anaphylaxis and symptoms which provide important information to the user in a convenient manner. Furthermore, it also includes a step by step training tool showing how to use EpiPen (an adrenaline auto-injector) tool to provide feedback on the correctness of the injection steps.

In higher detail, we reviewed the following existing applications that aim to support the eating experience of people with food allergies, including the user reviews. First, the biteappy application is a free mobile application for people with food allergy, food intolerances, and special diets (Biteappy 2018). One of its unique features is the translation feature that provides freeform phrases in over 30 languages. However, according to the user reviews, it lacks a pronunciation tool. Secondly, we reviewed AllergyEats, a mobile application that aims to help users to quickly find allergy friendly restaurants closest to their device location (AllergyEats 2017). Users reported that this application showed outdated restaurant information and few food allergies were supported. Additionally, we looked at AllergyBot, a chatbot application that aims to assist young adults with food allergies to find information about restaurants (Hsu et al. 2017). AllergyBot is built using instant messaging apps like Facebook messenger and displays information in an automatic manner. According to the user reviews of this application, they felt like they "were chatting to a friend". However, the participants reported that the layout was confusing and did not notice that some texts were clickable.

\section{ALLERGYFREEFOODIE: REQUIREMENTS, DESIGN AND EARLY IMPLEMENTATION}

Based on related work, we gathered our initial list of requirements for the AllergyFreeFoodie concept as follows: a) It should give instant recommendations for restaurants according to the user's location and allergies, b) It should enable rating and reviewing of restaurants, and $c$ ) It should provide a Chatbot that acts like Q\&A to provide information about the restaurants. Figure 1 and 2 illustrate early representations of the requirements throuah a series of low fidelity mock-ups.

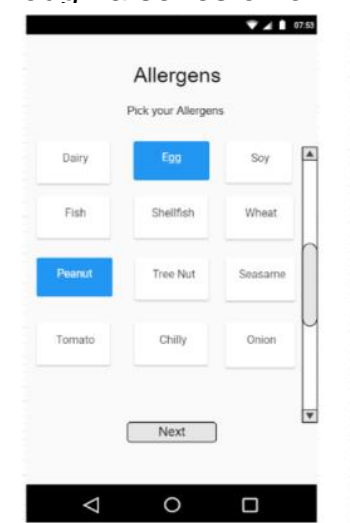

(a)

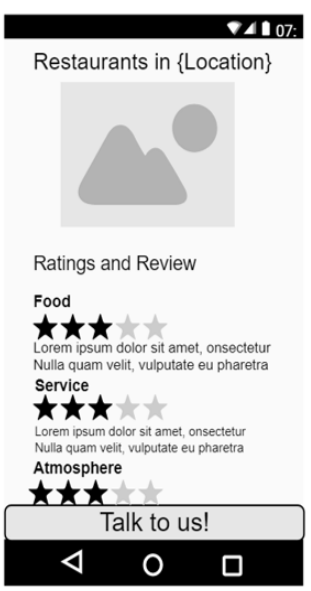

(c)

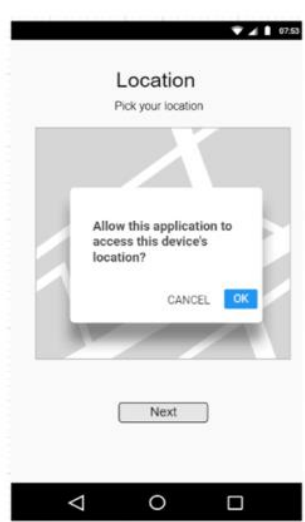

(b)

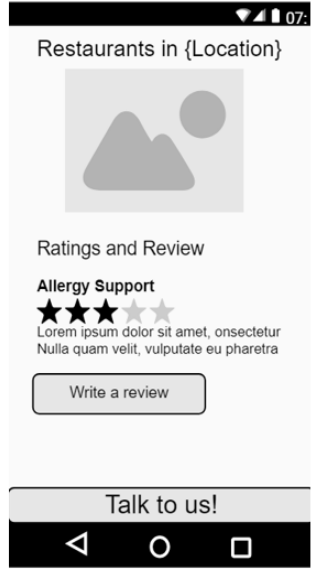

(d)
Figure 1: AllergyFreeFoodie functionalities: (a) select allergens, (b) select user location, (c-d) show restaurant's information and reviews 


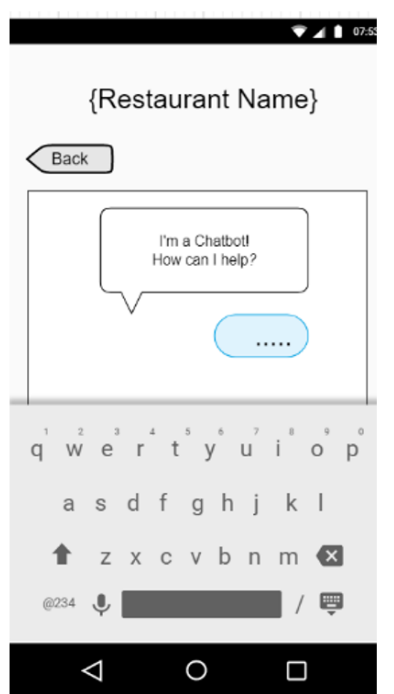

Figure 2: Chatbot functionality to create reservations, add reviews and a $Q \& A$ support for the user.

We envisioned and designed early mock-ups of a system where people with food allergies would be able to search for allergy-safe restaurants, cafes and other related food places. Users would start by entering their list of their own allergens (see Figure 1a). Then, users could select their location (see Figure $1 \mathrm{~b}$ ) and get access to a list of places where to eat around them including their customer reviews (see Figure 1c and 1d), based on parameters relevant for people with food allergies.

The application also has a chatbot (see Figure 2) which envisions the following features: creating a reservation, reminding users to add reviews and ratings, and a Q\&A support for inquiring the restaurant about their food allergy options.

Regarding its implementation, AllergyFreeFoodie is being built using React Native ${ }^{1}$ and Foursquare ${ }^{2}$ Application Programming Interfaces (APIs). It provides a list of restaurants that are closest to the user's location, with a customised menu and showing reviews and ratings in the current version.

\section{INITIAL EVALUATION AND EARLY FINDINGS}

In order to test the initial design of the application, we invited 6 participants who had various types of food allergies to be part of a focus group and gathered feedback on the AllergyFreeFoodie lowfidelity paper-based prototypes. The session was twofold. We started by opening discussion on how participants coped with their food allergies. Some of the questions asked: What type of food allergy do you suffer from? Do you use any kind of

\footnotetext{
${ }^{1}$ React Native is a framework for building native mobile apps. See https://facebook.github.io/react-native/.

${ }^{2}$ Foursquare is a social network for mapping out places. See: https://developer.foursquare.com/.
}

resources (for example websites/Facebook groups/forums/mobile apps) to support your selfcare management? Secondly, participants were asked to comment on the low-fidelity prototypes and discuss their features.

The participants were 25-40 years old and suffered from food allergies such as peanut, wheat, and/or egg. The primary members of their care network were parents, partners, and friends. When asked about the type of resources that they used, most of them said they have not seen the need to use technology. For example, a participant shared this:
"I suffer from peanut allergy ... I don't use any mobile applications or joined any Facebook groups I feel there isn't any technology out there that is helpful or reliable for my allergy also that supports my way of life".

Participants also mentioned that they always read the packaging information when trying out new food. One of the participants said:

"When I'm out and need a snack, I usually buy
something that I know is safe. If I buy something
I haven't tried before, I always check the labels."

When asked about how they cope with their food allergy most participants said they just avoid the contact with allergens in everyday life situations. For instance, a participant clearly explained the main challenges they faced while eating out.

\footnotetext{
"Going out to eat is one of the problems I face. I feel anxious ... because I am allergic to peanut and egg, I find it difficult to find dishes that I like, and I worry about how the food is prepared and the cross contamination, so I try to avoid going out."
}

While being outside the home, it turns out very difficult to negotiate and control the potential risks of allergic reactions that can impact their social relationships and activities. For instance, a participant shared:

\begin{abstract}
"When my husband and I are invited to a dinner at someone else's house, I usually tell them beforehand about my allergy. But there have been situations where the host forgot about my allergy or didn't take it seriously... I feel like people don't understand the consequences of a food allergy"
\end{abstract}

Regarding the mock-ups, participants found the focus of the application to be positive and unique as they have not come across a similar application before. However, half of them felt confused about the interface, for example, they were not sure how the chatbot would work. In addition, participants suggested the option to add their own types of allergies together with the level of severity (low to high) to the application. Participants suggested an 
"Add to favourites" option for dishes and/or meals that they appreciated eating regularly. Furthermore, they requested a way to sort the restaurants by cuisine or by distance. Finally, participants recommended including the option to "drag and drop" in the map, to search for restaurants that are away from their current location.

\section{DISCUSSION}

Similar to previous studies (Begen et al. 2018; Pitchforth et al. 2011), our findings show how eating out can be a challenging experience as expressed by our participants that also involves their care network. Food allergies might impact not only the way they interact with food but also their social relationships with family and friends. Regarding the application, participants highlighted the benefits of using it to search for restaurants that accommodate their needs. Participants asked to include the level of allergy severity into the application. Rather than selecting the types of allergies every time they open the application, they suggested to personalize it with their own types of allergies. Participants also mentioned the flexibility of the application to move the location around the map to filter options that can be distant from their current location.

\section{CONCLUSION}

In this paper we presented the AllergyFreeFoodie concept, a crowdsourced mobile application to support people with food allergies to find safe places for eating out. We gathered feedback on the initial design and its potential benefits. The next step will be to implement participants' suggestions and gather additional feedback. Our expectation is that AllergyFreeFoodie will support people's eating out experiences and enhance their current strategies to manage food allergies in everyday life. We aim to promote allergy-friendly environments and decrease the social barriers imposed to people with food allergies. Furthermore, we envision an application that can be adapted and adopted by food establishments to incorporate their menus on it, support people's information seeking practices and communication, recognizing the importance of accommodating food menus to customer's needs considering different types of allergies.

\section{ACKNOWLEDGEMENTS}

We thank all participants who shared their lives with us. FN acknowledges the financial support from North Portugal Regional Operational Programme (NORTE 2020), Portugal 2020 and the European Regional Development Fund (ERDF) from European Union through the project 'Deus ex Machina: Symbiotic Technology for Societal Efficiency Gains', NORTE-01-0145-FEDER000026.

\section{REFERENCES}

AllergyEats (2011) Find Restaurants - AllergyEats. [Accessed $04 \quad 15, \quad 2018]$ https://www.allergyeats.com/

Begen, F. M., Barnett, J., Barber, M., Payne, R., Gowland, M. H., \& Lucas, J. S. (2018). Parents' and caregivers' experiences and behaviours when eating out with children with a food hypersensitivity. BMC public health, 18(1), 38.

Biteappy (2018) Biteappy. Accessed 04 15, 2018. https://www.biteappy.com/

Davidson, N., Vines, J., Bartindale, T., Sutton, S., Green, D., Comber, R., Balaam, M., Olivier, P. and Vance, G. (2017) "Supporting Self-Care of Adolescents with Nut Allergy Through Video and Mobile Educational Tools." In Proceedings of the $2017 \mathrm{CHI}$ Conference on Human Factors in Computing System 1078-1092.

FSA (2016) Quarter of people with food allergies suffer reactions when eating out | Food Standards Agency [Accessed 04 08, 2018] https://www.food.gov.uk/news-

updates/news/2016/15103/quarter-of-peoplewith-food-allergies-suffer-reactions-when-eatingout

Henricksen, Karen, and Stephen Viller (2012) "Design of software to support families with foodallergic and food-intolerant children." In Proceedings of the 24th Australian ComputerHuman Interaction Conference 194-203.

Hernandez-Munoz, Luis U., Sandra I. Woolley, David Luyt, Gary Stiefel, Kerrie Kirk, Nick Makwana, Cathryn Melchior et al. (2017) "Evaluation of AllergiSense smartphone tools for adrenaline injection training." IEEE journal of biomedical and health informatics 21, no. 1272 282.

Hsu, Paris, Jingshu Zhao, Kehan Liao, Tianyi Liu, and Chen Wang (2017) "AllergyBot: A Chatbot Technology Intervention for Young Adults with Food Allergies Dining Out." In Proceedings of the $\mathrm{CHI}$ Conference Extended Abstracts on Human Factors in Computing Systems 74-79.

Jyonouchi, H. (2008). Non-lgE mediated food allergy. Inflammation \& Allergy-Drug Targets (Formerly Current Drug Targets-Inflammation \& Allergy), 7(3), 173-180.

Lee, Yee Ming, and Junehee Kwon (2011) "Exploration of Attitudes and Behaviors of Consumer with Food Allergies about Dining Out: A Focus Group Study."

Luyt, David, Heidi Ball, Kerrie Kirk, and Gary Stiefel (2016) "Diagnosis and management of food allergy in children." Paediatrics and Child Health 26, no. 7: 287-291. 
nhs.uk. (2016). Food allergy. [Accessed 04 15, 2018]https://www.nhs.uk/conditions/food-

allergy/.

Pitchforth, E., Weaver, S., Willars, J., Wawrzkowicz, E., Luyt, D., \& Dixon-Woods, M. (2011). A qualitative study of families of a child with a nut allergy. Chronic illness, 7(4), 255-266.

Sacla (2016) Eating Out Today and Tomorrow. [Accessed $\quad 04 \quad 15 \quad$ 2018]. http://www.fsc.uk.com/wpcontent/uploads/2016/11/SaclaReport-PDFFINAL.pdf 